\title{
Streamlining Ground Station Network Compatibility Test for Small Satellites
}

\author{
Scott H. Schaire ${ }^{1}$, Steven N. Bundick ${ }^{2}$, Christopher J. Roberts ${ }^{3}$, Leslie L. Ambrose ${ }^{4}$, Jerry L. Mason ${ }^{5}$, Serhat. \\ Altunc $^{6}$ and Yen F. Wong ${ }^{7}$ \\ NASA Goddard Space Flight Center, Greenbelt, MD 20771, USA
}

Mark R. Lamberson ${ }^{8}$ and Joe Baros Jr. ${ }^{9}$ Peraton Corporation, Greenbelt, Maryland, 20771, USA

Peter B. Celeste ${ }^{10}$ and Patricia R. Perrotto ${ }^{11}$

Booz Allen Hamilton, Annapolis Junction, MD 20701, USA

and Mark A. Bollard ${ }^{12}$

ARES Corporation, Greenbelt, MD 20770, USA

\begin{abstract}
A team of eight subject matter experts at NASA Goddard Space Flight Center (GSFC) completed a Lean Six Sigma project to identify process improvements for the compatibility test process for small satellites planning to use the NASA Near Earth Network (NEN). Ground station network compatibility testing is designed to reduce the risk to missions by resolving issues between the spacecraft's flight communication and navigation components and the ground systems prior to launch. Compatibility testing, which consists of a series of tests performed over a period of months and documented in reports, is an important step meant to prevent post-launch anomalies that could lead to expensive troubleshooting or mission failure. Compared to traditional missions, small satellite missions typically have a smaller budget and compressed schedules, which can result in small satellite projects' willingness to accept the risk associated with less comprehensive compatibility testing. Optimization and or refinement of the compatibility test process for small satellite missions could alleviate some of the pressures inherent with these factors. The goal of the Lean Six Sigma project was to develop alternative scalable methods of compatibility testing for small satellites. The Lean Six Sigma approach and the results of the project are reviewed in this paper.
\end{abstract}

\section{Introduction}

\footnotetext{
$\mathrm{T}$ he NASA Near Earth Network (NEN) is comprised of stations distributed throughout the world. NEN services are provided through NEN-owned and operated ground stations, Partner agencies (e.g., National Oceanic and Atmospheric Administration (NOAA) Command and Data Acquisition (CDA)), and Commercial ground station

${ }^{1}$ NASA Near Earth Network (NEN) Wallops Manager, NASA Near Earth Network (NEN).

${ }^{2}$ Senior RF Engineer, NASA Wallops Electrical Engineering Branch.

${ }^{3}$ Mission Manager, Exploration and Space Communications Division, ALAA Member.

${ }^{4}$ Networks Integration Manager, NASA GSFC Networks Integration Management Office.

${ }^{5}$ Branch Head, NASA GSFC Telecommunication Networks \& Technology Branch.

${ }^{6}$ NEN Upgrade Manager, Telecommunication Networks \& Technology Branch.

${ }^{7}$ Senior RF Engineer, NASA GSFC, Telecommunication Networks and Technology Branch.

${ }^{8}$ Systems Engineer III, NEN Operations.

${ }^{9}$ Compatibility Test Engineer/Technical Lead, Network Engineering and Integration.

${ }^{10}$ Senior Lead Engineer, NASA Integrated Management Team.

${ }^{11}$ Lead Engineer, NASA Integrated Management Team.

12. Sr. Systems Engineer, Quality Engineering Branch, GSFC Lean Six Sigma Program Office.
} 
providers (e.g., Kongsberg Satellite Services (KSAT), Swedish Space Corporation (SSC) and its subsidiaries). The Near Earth Network Project

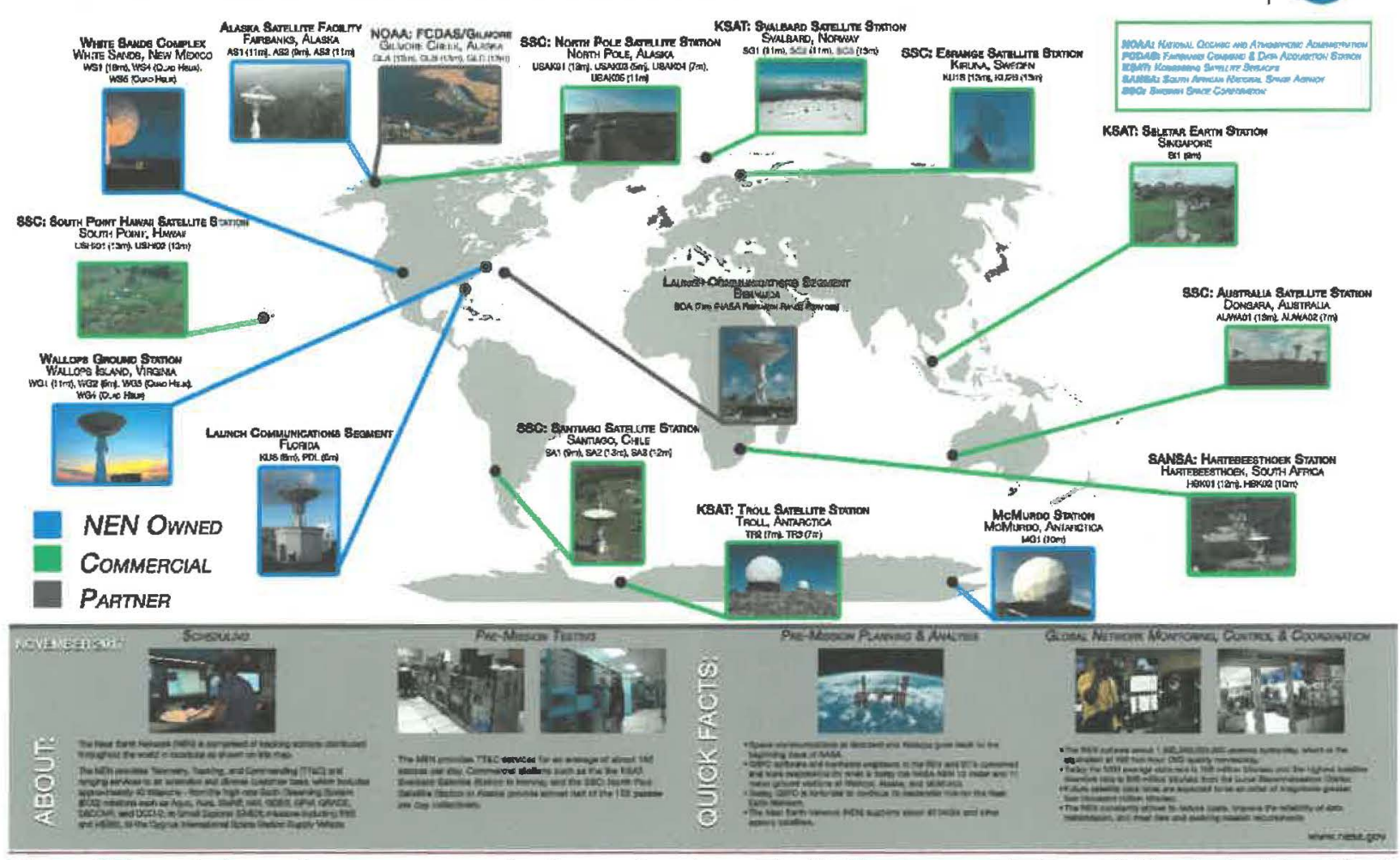

Figure 1Figure 1 shows the current network of ground stations. The NEN supports missions orbiting in the Near-Earth region, from Earth to 2 million kilometers. Communication services are provided for various low-Earth orbits (LEO), geosynchronous orbits (GEO), highly elliptical orbits (HEO), LaGrange orbits, lunar and suborbital, and launch trajectories. 


\section{The Near Earth Network Project wam}

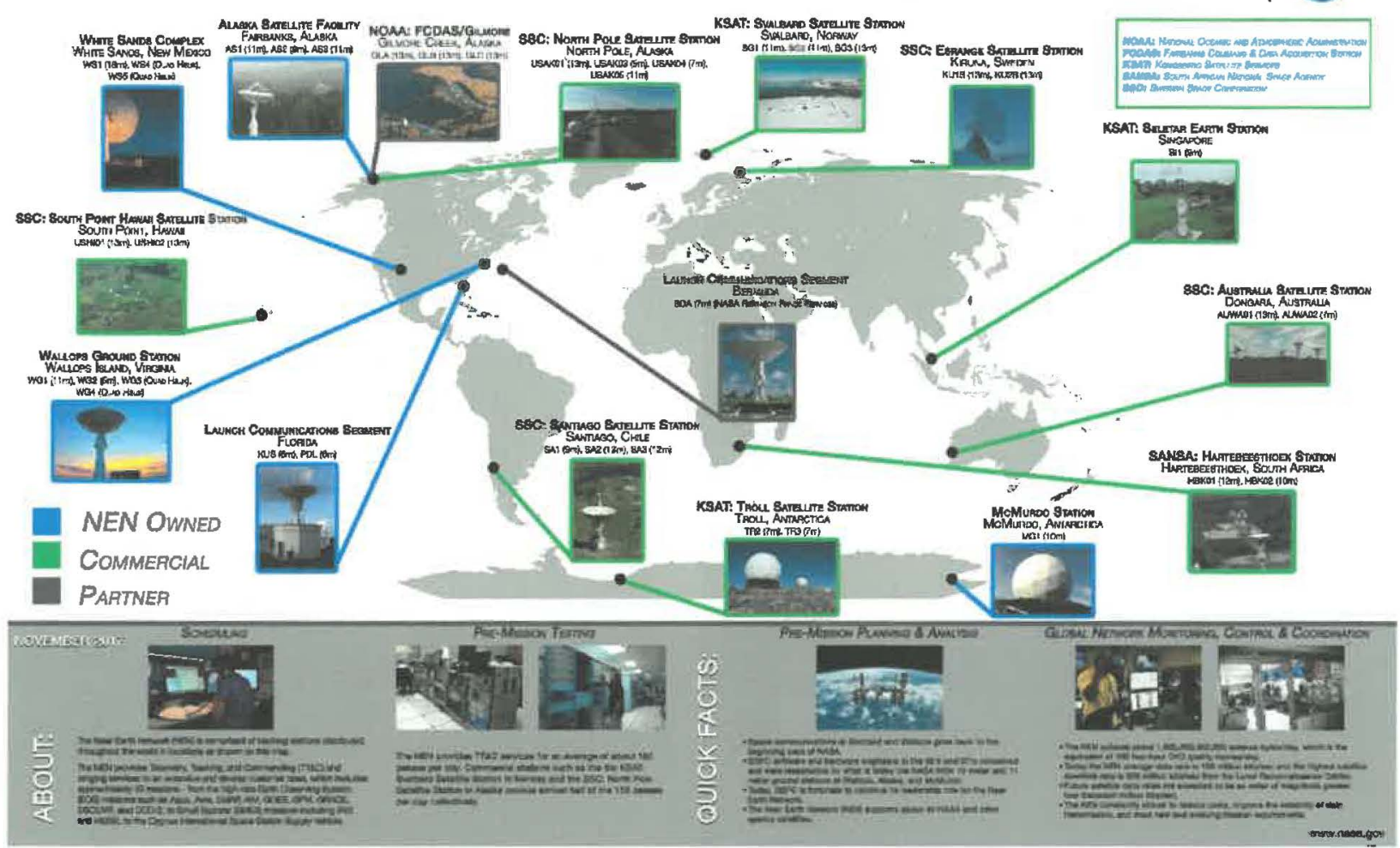

Figure 1 The NEN Supports Orbits in the Near Earth Region from Earth to 2 Million Kilometers

The NEN has historically provided support to traditional satellite missions; however, in recent years the NEN has begun to be considered as the primary or secondary support network by small satellite projects.[1]

The first CubeSat, a sub-classification of small satellites, to fly baselining NEN support will likely be the SeaHawk CubeSat. SeaHawk is scheduled to launch in 2018 and is being developed as part of the Sustained Ocean Color Observations using Nanosatellites (SOCON) project. Like many other CubeSats, it is a proof of concept for a potential larger group of identical CubeSats taking multiple simultaneous earth science measurements and reducing the revisit time to any one spot on earth. SeaHawk represents the future wave of small satellite missions to require use of the NEN.

In LEO orbit, a CubeSat with a $1 \mathrm{~W}$ X-band transmitter and a $5 \mathrm{~dB}$ gain antenna could achieve a $130 \mathrm{Mbps}$ data rate with the NEN, subject to National Telecommunications and Information Administration (NTIA) bandwidth restrictions. A CubeSat in the same orbit with a $1 \mathrm{~W} \mathrm{S-band} \mathrm{transmitter} \mathrm{and} \mathrm{a} 0 \mathrm{~dB}$ gain antenna could achieve a 500 kbps data rate with the NEN with significant margin. Compatibility testing combined with link analysis will be critical for validating these data rates.

\section{Today the NEN is capable of supporting small satellites including CubeSats with S-band, $X$-band and Ka-band services.}

The objective of the NEN is to provide services of sufficient type and quality to meet requirements for NASA missions, as efficiently (inexpensively) as possible. The NEN is exploring ways to better support small satellites, which typically have a fraction of the budget and are operating on compressed schedules when compared to larger traditional satellite missions. One initiative is to explore methods to reduce the cost of ground network services for small satellite missions to better align with their smaller budgets. 
The incremental recurring costs for missions using NEN with NEN-owned stations is negligible, because the NEN is highly automated. Most of the labor effort to integrate a new mission into the NEN is associated with Mission Planning, Integration, and Test (MPI\&T) functions, which include coverage, loading, and feasibility analyses; radio frequency (RF) analyses; compatibility testing; and end-to-end testing. This paper focuses on concepts for reducing the cost of compatibility testing for small satellites based on the results of a Lean Six Sigma project.

\section{Project Assumptions}

Figure 2 shows an increasing slope that indicates the growth of CubeSats from 2000 to 2015. It is a graph of cumulative number of CubeSats launched by organization [2]. The National Academies of Sciences report mentions that "small satellites are gaining momentum to answer science questions in a rapid and possibly more affordable manner." The number of CubeSat launches is predicted to increase substantially, in part due to NASA launch initiatives (e.g., CubeSat Launch Initiative (CSLI), Exploration Mission (EM) flights). The NEN recognizes the important role small satellites, including CubeSats, are beginning to play in carrying out scientific missions and that they could constitute a significant shift in the NEN-supported mission set within the foreseeable future.

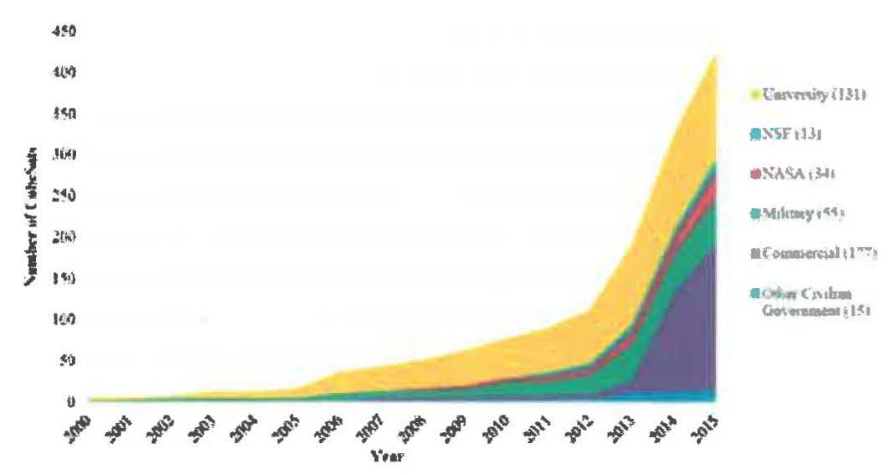

Figure 2 CubeSat Launch Trend

The Goddard Space Flight Center (GSFC) Networks Integration Management Office (NIMO) is responsible for MPI\&T for the NEN. MPI\&T includes compatibility testing. In the Spring of 2017, the NEN and NIMO worked together on a Lean Six Sigma (LSS) project to examine and propose compatibility test process changes that will enable NIMO to be more responsive and cost effective for small satellite missions.

Compatibility testing assesses the RF interface between the spacecraft and the NEN prior to launch with the goal of exposing ground station equipment configuration errors or spaceflight hardware issues. This testing is conducted at the GSFC Compatibility Test Laboratory (CTL) or at the customer's facility using compatibility test equipment in transportable/shippable equipment racks. The traditional NIMO mission integration approach is documentation and testing intensive to ensure that the network's customers can achieve communications connectivity with minimal risk.

It is feasible for small satellites to bring their satellite to GSFC or the ground station rather than requiring tests at the customer's facility. CubeSat RF compatibility testing has become more streamlined already. Since CubeSats are inherently small, it is practical to bring the spacecraft itself to a ground station and test. In reality this is the ultimate RF compatibility test since the spacecraft is being tested with the exact equipment that will be used for on-orbit support. Typically, the CubeSat RF interface is less complex than the RF interface for a larger, traditional satellite as well. There is usually a single downlink mode, uplink mode and data rate, and no metric range or Doppler tracking requirements. This keeps the number of tests to a minimum, reducing personnel and travel costs.

Small satellites, including CubeSat missions, may be willing to accept more risk in return for reduced testing costs. The NASA Wallops Flight Facility has an 18-meter Ultra-High Frequency (UHF) half duplex ground station, which is outside the NEN, that is being used to support CubeSat missions. Being outside the NEN, there was no pre-existing process for UHF compatibility testing. Rather than the traditional method of using off-line equipment for testing, to date, users have brought their fully configured CubeSat to the ground station for RF compatibility testing. Testing generally consists of open loop uplink command and downlink telemetry transmissions. Open loop testing is necessary in many cases since the satellite is usually fully configured for launch at this point in the schedule, and disconnecting the antenna or cables from the transceiver is not desired. Open loop testing is conducted in one of two methods. The 
first method is to place the CubeSat on a building roof approximately one mile from the ground antenna. The uplink and downlink is tested through the entire ground terminal. The ground system power amp must be attenuated, and power out of the spacecraft transmitter may be reduced as well, if practical. A second method is to test with the spacecraft in the ground system operations room. A stub antenna is connected to the ground station RF front-end equipment, and open loop testing is conducted in this manner. This type of risk mitigation testing typically takes place over the course of about one day with minimal documentation and results in finding communication issues prior to launch, reducing risk. This type of testing is effective, even though it is very low cost and the tests are truncated.

The goal of the LSS Project was to improve efficiency and develop lower cost options for implementing compatibility testing, particularly for small satellites, including CubeSats. The team identified weaknesses in the current processes and equipment and developed recommendations for modifications and enhancements to processes and equipment. As a result of productivity improvements, the team will enable support for a greater number of missions without additional staff.

Some of the recommendations of the team only apply to small satellites. It was found early in the LSS Project, that improvements for small satellites will also benefit sounding rocket, balloon, and unmanned aerial vehicle (UAV) missions. Other changes will reduce cost and schedule for all compatibility tests.

Starting with a baseline cost and schedule for testing at GSFC, the team set ambitious targets of achieving a schedule reduction of greater than $75 \%$ and a cost reduction of greater than $80 \%$. The end result was that a combination of recommendations, if implemented, would reduce costs by greater than $60 \%$ and significantly reduce schedule.

There are typically four to ten compatibility tests run per year for NEN for all missions, including small and large satellites. Streamlining options identified can all co-exist; multiple solutions are possible. The implementation of streamlining options is constrained by limited funds available for investment. GSFC is in the process of implementing the recommendations, as funds allow, on a non-interference basis.

\section{LEAN SIX SIGMA}

LSS is NASA's structured approach to continuous improvement. NASA embarked on their LSS journey as a grassroots endeavor initiated at the Marshall Space Flight Center (MSFC) in 2004. Since that time, each of the ten NASA Centers, along with NASA Headquarters (HQ), has developed a Lean Six Sigma Program Office to encourage and facilitate the use of LSS methods and tools for their unique continual improvement efforts.

NASA has integrated the concepts of Lean with Six Sigma to enable a robust process improvement effort regardless of the "type" of problem or issue to be resolved [3], incorporating the use of teams.

\section{A. Lean - The pursuit of Waste Elimination}

The concept of Lean entails identifying and eliminating all non-value-added activities, simplifying work processes, and increasing efficiency [4].

1) Specify what creates value from the customers perspective

2) Identify all the steps along the value stream

3) Make those processes flow

4) Make only what is necessary and pulled by the customer

5) Strive for perfection by continually removing waste.

\section{B. Six Sigma - The pursuit of Process Perfection}

The concept of Six Sigma is focused on reducing variation in a process and eliminating defects.

1) Data is collected to analyze processes

2) Enables data driven decision making

3) Uses facts and data to reduce variation.

\section{The Use of Teams}

"Teamwork is the ability to work together toward a common vision. The ability to direct individual accomplishment toward organizational objectives. It is the fuel that allows common people to attain uncommon results." Andrew Carnegie

Teams of employees are critical to the success of any LSS team. NASA applies teams of subject matter experts to LSS teams to create those "uncommon results" as Andrew Carnegie referenced. In addition to the team members, each team has a defined team leader. The team leaders are also subject matter experts and may have received training in LSS tools and processes. Each LSS team is also facilitated by a LSS expert, either a Certified Black Belt, or Green Belt, or LSS candidate for Green Belt certification. The role of the facilitator is to support the team through the Define, Measure, Analyze, Improve, and Control (DMAIC) process, to provide expertise in the use of LSS tools and to enable the team to achieve the overall objectives of their improvement activity. These facilitators are proven subject matter experts in the LSS tools and methodologies. 
The NEN/NIMO LSS Team was uniquely qualified to effectively execute this LSS Project. The team was composed of both civil servant and contractor personnel that embodied all of the technical expertise and experience relevant to driving efficiency within CubeSat RF compatibility testing activities. The team also included individuals that had received training in LSS processes, methods, and tools. The team leader, S. Schaire, had recently attended NASA's 40-hour Lean Six Sigma Green Belt Level Certification training. Additionally, C. Roberts had participated in a NASA Just-in-Time LSS Green Belt Level Certification project to increase the efficiency within the NEN Launch Communications Segment (LCS). Combined, these two LSS practitioners were able to provide additional support and guidance throughout the team's activities.

At NASA all LSS projects are initiated with a Charter - a two-page document defining the purpose, scope, team objectives, preliminary project plan and other team information necessary to effectively achieve the objectives outlined in the charter. This team was no different.

\section{DMAIC-A Structured Approach to Continual Improvement}

As noted above, LSS employs a structured and disciplined approach to continuously improve products, processes, and services. See Figure 3 for a detailed description of the five-Phase methodology. The five-phase methodology employed in a LSS improvement project is referred to as Define, Measure, Analyze, Improve and Control (DMAIC) process [5]:

1) Define - Identify the project objectives and customer (internal and external) deliverables.

2) Measure - Measure the process to determine current performance; quantify the problem.

3) Analyze - Analyze and determine the root cause(s) of the defects/variation/waste, $\mathrm{Y}=\mathrm{f}(\mathrm{x})$.

4) Improve - Improve the process by eliminating defects/variation/waste per project objectives.

5) Control - Control future process performance, institutionalize the improvement, and ensure ongoing monitoring.

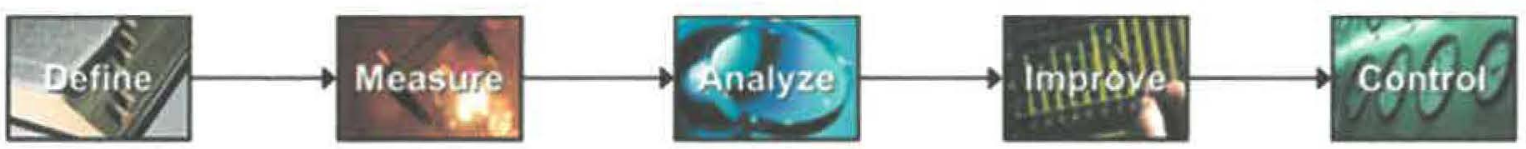

Activities

- ifensay Problen

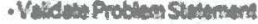

- Gether toc of the

Customer s lusinass

- Valdute Goustament

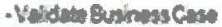

- Vaidat Propea Seape

- Select andLunch Tears

- Oeviop Projed Schedus

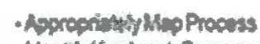
- benrily key hout procass end atoul Wetics

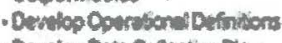
- Devwicosecta Coluction Plon - Yolded lisasurement Sysions - Collinat Busuline Ota - Devervias Precess Purbormencepousty
- Lenity PotentiarootCuns

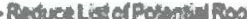
Cevess

- Confins roct Cuna boutous Relutontis

- Estrmat fromet of RodCuas enkry Outoss - Pronita Root Causes
- Orvepp Patentis Solution

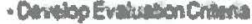
- Select Best Solutions - Conwop To te Processulap(y)

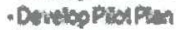

- Pilor Schuton

- Drvesos Full Scile implomerestonfien
- Inplemera Mistroteprocling

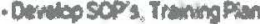
nd Prosess cantods - Implimesi Soltion and Ongoing Proces: shesourements - dontsprojuct Regiention Opportunios

- Tranedion Project to Process Owner

\section{Tools}

- Projed Chintar

- Projeci Solueson Toos

- Vaus Sirem 4 o

- Veroun fineno intidos

- Elnetive unowroskils

- Senivertelder Analysis

- Commencivonplan

- Sipoc miep

- Nighlend Procens ke

- Projuct hentenmint Took

- Voc end kreno And bs

- Racl and Qued Churts

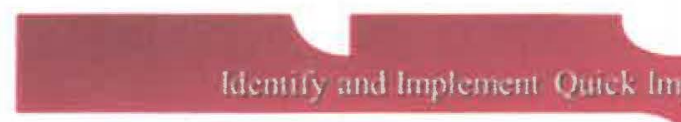

Ideniify and Implement Quick Improvements with Kaken

- Process lutepiro

- Process Cjola Etciency

- Litiols Len

- Operatentowindors

- Dats colluction Pian

- Stebistear Sumpling

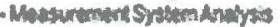

- TPen

- Generiepul

- Sulup Ruduction

- Consel Cherts

- Hatoperans

- Constrinildenticertion

- Process cenpability
- Vatue Andy

- Prociess conatrinto

- Tekt Tine Andryis

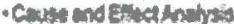

- FMEA

- Hypethesis Tess

- Cometion Anetris

- Simpia end Multople Regresaich

- MoVA

- Compenanter alvarition

- Conquarrin Produd erd

Processs Complexify

- Ouweng Thiony

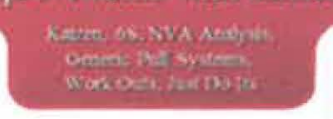

- Breindoresting

- Rspluniatrrent PulKarben

- Stacking Stringy

- Process 7 row moroviment

- Process betencine

- An dicel Buter Sitino

- Total Produetire Rantirsence

- Designel Experienents roc5

- Solutar Selection Mitria

- To-Se'Process Mapping

-Poks-Yok

Figure 3 Roadmap for a Lean Six Sigma Project - All NASA LSS Projects Follow the 5-Phase DMAIC Process. Activities and Tools are tailored for each Project

As with all NASA LSS teams, the NEN/NIMO LSS Team followed the DMAIC process. The activities and tools applied within each phase of the DMAIC process is pre-defined and tailored for each project and the team is afforded flexibility to modify the activities throughout the project as events warrant and needs change. 
The NEN/NIMO LSS Team initiated their project through the use of a Team Charter that was developed by the team leader and LSS Black Belt. Based on discussions with the team leader, the LSS Black Belt defined this LSS Project as a Kaizen project [6]. Within the NASA Lean Six Sigma Program, Kaizen projects are a

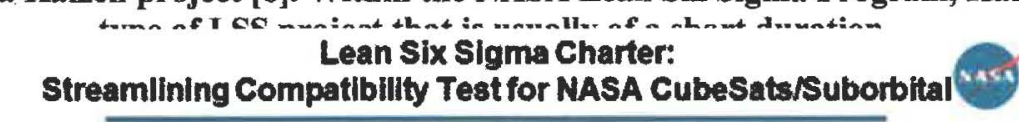

\begin{tabular}{|c|c|}
\hline \multirow{4}{*}{ 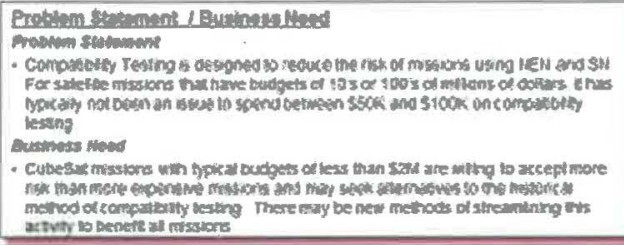 } & 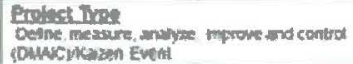 \\
\hline & Syem Dathe \\
\hline & Possibly in lanen, $2017 \mathrm{Nat}$ on Fildays. \\
\hline & \multirow{2}{*}{ 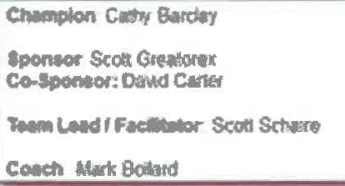 } \\
\hline 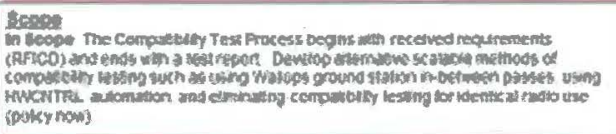 & \\
\hline 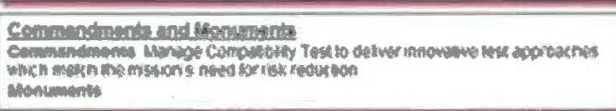 & \multirow{2}{*}{ 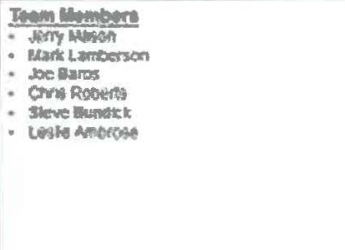 } \\
\hline 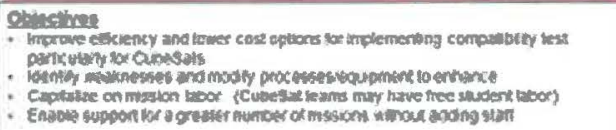 & \\
\hline
\end{tabular}

Figure 4Figure 4 shows the Charter for this team.

\section{Lean SIx Sigma Charter:
Streamlining Compatibility Test for NASA CubeSats/Suborbital $($ Ast}

\begin{tabular}{|c|c|}
\hline 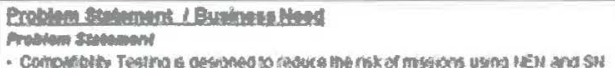 & 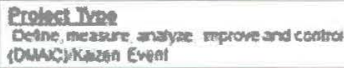 \\
\hline 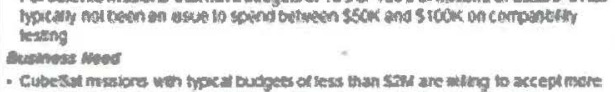 & 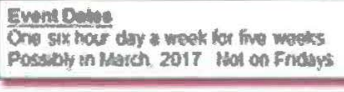 \\
\hline 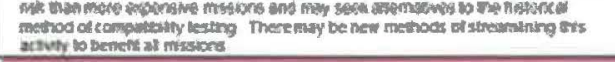 & \multirow{2}{*}{ 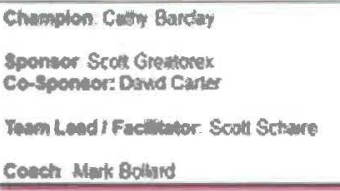 } \\
\hline 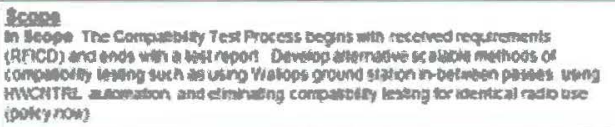 & \\
\hline 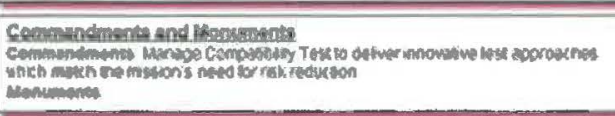 & \multirow{2}{*}{ 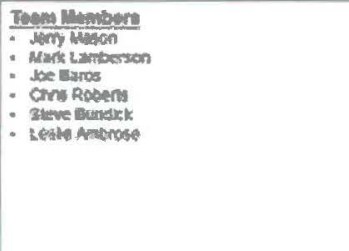 } \\
\hline 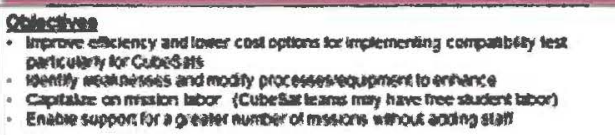 & \\
\hline
\end{tabular}

Figure 4 Lean Six Sigma Charter; Required to Initiate any Lean Six Sigma Improvement Project

\section{E. Building the Streamlining Implementation Plan}

LSS teams typically start their first team meeting with what NASA refers to as an in-brief or kick-off. The purpose of this 30-minute briefing is to brief the entire team on the purpose, scope, objectives, and goals as well as the 
Preliminary Project Plan. Following suit, the team began the effort with an in-brief. The LSS Black Belt prepared and delivered the in-brief to the team. Based on the composition of the team, the LSS Black Belt included very short tutorials on a few of the LSS tools that would be utilized by the team. These short tutorials included material addressing developing a supplier, input, process, output and customer (SIPOC) matrix, rules and process for brainstorming, developing a "swim-lane" process map, and conducting a "value-added" analysis. This briefing enabled the Black Belt facilitator to reference these tools throughout the day, ensuring all team participants have a basic understanding of the activities and can effectively participate. The in-brief also provided team members an opportunity to ask questions. Many team members may not understand their role on the team and most members have a very limited knowledge of LSS going into the events. Of the entire team, only one individual had been certified as a NASA LSS Green Belt and the Team Leader, had recently attended NASA's 40 hour Lean Six Sigma Green Belt Training. Also on the team was a NIMO representative, who is the primary interface between NIMO and Space Science customers. The team included subject matter experts (SME) in the fields of ground station and spaceflight communication system design and test. The team members had the knowledge, skills and abilities to examine the NEN compatibility test activities from a process perspective.

Each team meeting was conducted with a structured agenda, specific activities, and purpose. The team began the project by defining and documenting the current process for conducting compatibility testing. Figure 5 shows the small team working on documenting the existing compatibility test process.

Once the process was defined and documented, the team identified non-value-adding activities. The team labeled process steps as Green ("value-added"), Yellow ("non-value added, but required") and Red ("non-value added").

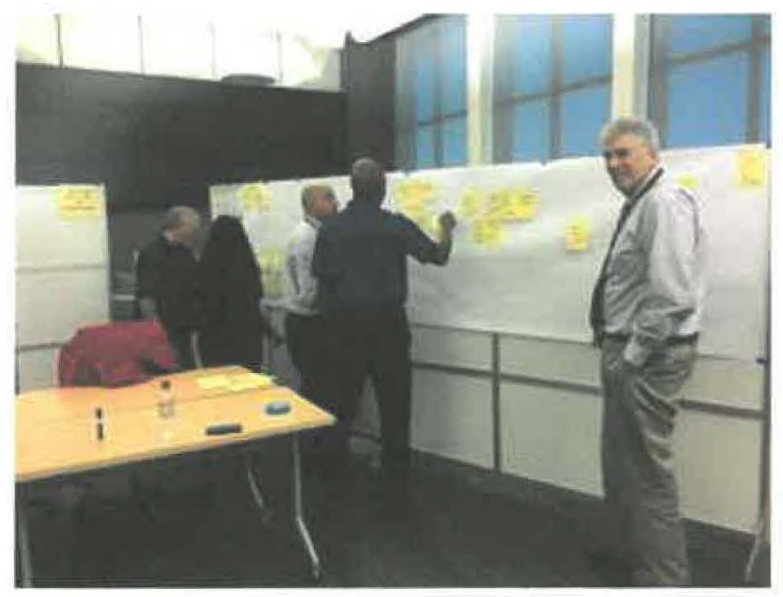

Figure 5 Compatibility Team Mapping the Current Process for Compatibility Test Process

The "value-added" analysis that is referenced in the prior paragraph can be the most difficult for any team to perform. The SME's on the team are often the individuals performing the process steps and to label an activity as "non-value added" can be difficult for many individuals. The LSS Black Belt encouraged objective, process-focused discussions, and an understanding that although a process step may be labeled as "non-value added", that does not mean the process step will be removed. It does however, provide a signal to the team for additional analysis and discussion.

Considering the baseline process also led the team to focus on the parts of the compatibility test process before and after the actual testing (e.g. planning, report writing). The team evaluated the process steps that according to LSS methods were not "value-added". In order for a process step to be counted as "value-added", it must meet all of the following criteria:

1) Customer willing to pay

2) Done right the first time

3) Changes the product or service delivered.

The team found that there were a number of steps that were not "value-added" or bordered on not "value-added". Some of these steps were related to verification of the test equipment configuration, and reviews of test results and reports.

At the conclusion of the "value-added" analysis activity, brainstorming sessions were conducted to identify problems, issues, barriers, and other negative impacts that may be causing the process to not achieve optimal 
performance (and in this instance - higher costs). Once the list of "issues" was developed, edited and clarified, it was posted for all team members to see.

Using the "issue" list as a guide, the team embarked on follow-up brainstorming sessions to identify improvements to the process. The team identified over seven different improvements to the process. Based on this broad list of improvements, the team applied a Possible, Implement, Challenge, and Kill (PICK) chart analysis to prioritize their solutions. The PICK is a quad chart with "Ease of Implementation" along the X-axis and "Level of Impact" along the $Y$ axis. The team mapped their solutions onto the PICK chart. This step refined the alternatives for the team's final analysis. The team's final analysis, including impact, costs and implementation constraints, resulted in multiple potential improvements for compatibility testing resulting in recurring cost savings compared to conventional methods.

The team then built an Implementation Plan. The Implementation Plan documents what actions will be performed, and who will perform the actions. This plan, along with a formal out-brief was provided to the LSS Champion (management personnel who allocated resources and is in favor of conducting the project) and Sponsor (process owner). The team has since begun implementation with the expected results as noted throughout this document.

\section{Current Process/Baseline}

NIMO connects small satellite missions to the communications services and resources of the NEN. The process is initiated in the early stages of a mission with the development of a Customer Questionnaire to define the high-level network requirements. Based on these inputs, a Network Feasibility Analysis (NFA) is performed to assess the network coverage and loading capacity, and an RF Analysis is conducted to ensure that each mission will successfully establish connectivity with the NEN assets providing support. This RF Analysis is documented in an RF Interface Control Document (RFICD). The RFICD is a bi-lateral document signed by a representative of the NEN and the customer and used as the basis of compatibility testing, station configurations, and successful communication.

The network has simplified the requirements documentation for small satellites to both reduce cost and accommodate their shorter implementation schedule. Technical Interchange Meetings and Working Groups provide the forums to receive feedback from the customer and the network and refine mission requirements for the development of the Service Agreement and Requirements Document (SARD). The SARD commits space communications and data system services to be integrated by the NASA GSFC Exploration and Space Communications Projects Division (ESC) and defines the mission service requirements between the customer and the NEN.

The RF parameters defined in the RFICD as well as the network interfaces and requirements committed to in the SARD provide the baseline in the development of the Compatibility Test Plan. A kick-off meeting with the compatibility test team is coordinated when the RFICD and SARD have reached sufficient maturity to begin test planning. The current compatibility test process flow is depicted in Figure 6.

With an understanding of the mission network interfaces and requirements, funds for the test are initiated with a rough order of magnitude (ROM) estimate that is submitted to the Project for review and approval. The ROM includes assumptions and special hardware/software that is required beyond the normal support items needed to accomplish the test. Upon funding approval, a Lead Compatibility Test Engineer (CTE) is assigned to support test planning, test execution, and final reporting. In parallel, compatibility test splinter meetings are scheduled with prelaunch test representatives, the RFICD is received/reviewed, a test plan is drafted, and coordination begins with NEN representatives. During the splinter meetings, which are typically held by teleconference, the test plan progress is reviewed and updated. Test environment items, such as equipment interfaces, floor layout, and facility logistics, are also discussed with the project. The NEN representative confirms software and hardware versions and coordinates the loan of ground station loaner equipment. Ground station equipment is tested, scanned, and then shipped to the GSFC Compatibility Test Area (CTA).

Once the draft test plan is mature, it is distributed to the splinter test group for review. Comments are discussed with the reviewers, then when agreed upon, are incorporated into the final copy. The final version of the test plan is distributed to the prelaunch test team (i.e. multiple organizations involved in the mission) and management and filed in the NIMO online library. During the test planning period, CTEs integrate the NEN station equipment with the Compatibility Test System (CTS) (i.e. system under test) at GSFC. CTEs with years of experience and references to previous equipment configuration files, begin to set up parameters on loaned station equipment and the CTS that will be required for the compatibility test. One of the significant setup parameters for the ground station equipment is the loop bandwidth setting for each data rate based on the maximum Doppler shift and rate that is expected. Following pre-tests from GSFC/CTA the CTS system is packed and shipped, if testing is at a remote facility. Prior to the test 